Archive for

Organic Chemistry

Arkivoc 2020, part ii, 9-19

\title{
Mass spectrometric fragmentation of di-, tri-, and hexaquinane-fused dimethyl muconates induced by single and double electron ionization
}

\author{
Marco Harig and Dietmar Kuck* \\ Department of Chemistry, Bielefeld University \\ Universitätsstraße 25, 33615 Bielefeld, Germany \\ Email:dietmar.kuck@uni-bielefeld.de
}

Dedicated to Professor José M. Riveros on the occasion of his $80^{\text {th }}$ birthday

Received 10-08-2019

Accepted 12-03-2019

Published on line 12-17-2019

\section{Abstract}

The electron ionization mass spectra of nine muconic acid dimethyl esters that bear up to three muconate groups fused with a centropolycyclic di-, tri- and hexaquinane framework exhibit dominating fragmentation by loss of a methoxycarbonyl radical, ${ }^{\circ}$ COOMe, from the molecular radical cations, $\mathrm{M}^{\bullet+}$. Pyrylio-annelated structures for the $[\mathrm{M}-\mathrm{COOMe}]^{+}$ions are postulated in analogy to literature reports on simple muconic acids esters. The congeners bearing two or three dimethyl muconate groups lose also two ${ }^{\circ}$ CoOMe radicals after twofold ionization. The doubly charged fragment ions $[\mathrm{M}-2 \mathrm{COOMe}]^{2+}$ are proposed to contain two isolated pyrylium rings fused to the original tri- or hexaquinane core, thus representing polyaromatic distonic dications.

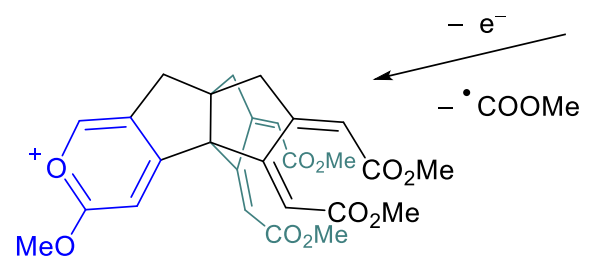

$m / z 511(100 \%)$
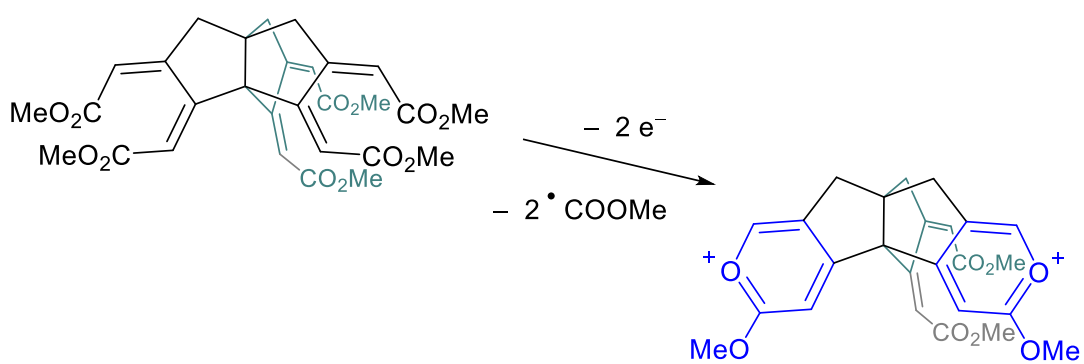

$m / z 226(25 \%)$

Keywords: Muconic acid esters, cyclization, pyrylium ions, dications, polyquinane derivatives, stereoisomers 


\section{Introduction}

An important sub-field of mass spectrometry has been called "Organic Mass Spectrometry" for more than half a century. ${ }^{1}$ This term reflects the exciting interplay of physical laws and chemical rules that can be recognized from mass spectra of organic compounds and their fragmentation behavior in a mass spectrometer. One of the great joys being an organic mass spectrometrist and, in the same time, an organic chemist who uses mass spectrometric analysis for the characterization of new or even novel organic compounds is, by looking at their mass spectra, to recognize surprising spectral features and, from these, to eventually gain deep insights into the chemistry of gaseous ions. ${ }^{2}$ Nowadays, this virtue has largely faded away in most laboratories that run mass spectrometric instrumentation - for various reasons. One of them is, of course, the overwhelmingly increased importance that mass spectrometry has found for bioorganic chemistry, biochemistry and medicinal application. Another, less obvious reason consists in the fact that contemporary ionization methods used for mass spectrometric analysis have turned from rather chemically pure to rather chemically impure techniques - and thus more difficult-to-control - reaction conditions. To be concrete, standard electron ionization (EI), being an energetically harsh but chemically well-defined technique to produce molecular radical cations, has lost importance as compared to modern ionization methodology, such as electrospray ionization (ESI) and matrix-assisted laser-induced desorption ionization (MALDI). These methods are well-known to be energetically mild but should be considered chemically much more complex, and complicated, than El.

This paper goes back to organic ions generated by El, i.e., in the absence of any matrix, from rather unusual organic molecules. After evaporation from a so-called solids probe into the ionization chamber of a conventional sector-field mass spectrometer, the molecular radical cations, $\mathrm{M}^{\bullet+}$, can be detected by the molecular ion peak at the proper molecular-mass $m / z$ value, and some mostly prominent peaks indicate the characteristic fragmentation reactions that may reflect the constitutional and, in many cases, also the stereochemical properties of the organic compound. Based on some compounds that originate from our research work on unusual three-dimensional, polycyclic-polyaromatic compounds, ${ }^{3}$ we would like to illustrate the joy with and the beauty of organic mass spectrometry based on this chemically pure - and somewhat puristic - way of acquiring mass spectra of organic compounds.

\section{Results and Discussion}

The compounds studied here were synthesized by ozonolytic degradation of a number of veratrole-based centropolyindanes of different molecular size with the original aim to explore an experimental access to a hitherto elusive parent polycyclic hydrocarbon, centrohexaquinane, $\mathrm{C}_{17} \mathrm{H}_{24}{ }^{4}$. The ozonolytic degradation process converted the veratrole units into the corresponding dimethyl cis,cis-muconates, while the structure of the bi- or polycyclic core was preserved. In the following, we first present and discuss the characteristic loss of a methoxycarbonyl radical, [MeO-C=O] $(59 \mathrm{u})$, from the molecular radical cations of the dimethyl muconates, thus providing interesting examples for this long-known El-induced fragmentation channel of muconic acid methyl esters and their diethyl derivatives. ${ }^{5,6}$ Then, as an intriguing and characteristic feature, the fragmentation by loss of two methoxycarbonyl radicals via doubly charged ions is presented.

The El mass spectrum of the diquinane-fused dimethyl cis,cis-muconate $\mathbf{1}$ is shown in Figure 1 . It essentially exhibits an intense molecular ion peak at $\mathrm{m} / \mathrm{z} 358(70 \%)$, minor peaks for the loss of a methoxy radical $(\mathrm{m} / \mathrm{z} 327,6 \%)$ and the elimination of methanol $(\mathrm{m} / \mathrm{z} 326,10 \%)$, and the base peak indicating the loss of a methoxycarbonyl radical $(\mathrm{m} / \mathrm{z} 299,100 \%)$. We assume that the latter process occurs by cyclization and 
subsequent allylic C-C bond cleavage, in analogy to the fragmentation of dimethyl muconates as well as benzylideneacetones, cinnamic acids and cinnamic aldehydes discussed in the literature. ${ }^{5-11}$ The elimination of methanol should take place by $1,5-\mathrm{H}$ transfer of a hydrogen atom from one of the allylic methylene or methine groups of the diquinane moiety of the molecular ion $\mathbf{1}^{\bullet+}$. The low relative abundance of the $[\mathrm{M}-\mathrm{MeOH}]^{\bullet+}$ ion is in accordance with the all-cis-stereochemistry of the muconate grouping which requires cis-trans isomerization prior to the 1,5-H transfer step. The major fragmentation path leading to the loss of either of the

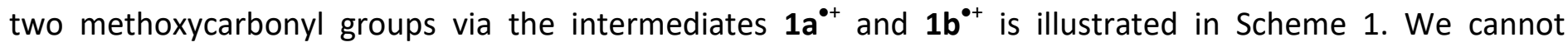
distinguish between these two similar routes without appropriate isotope labeling experiments. However, it is

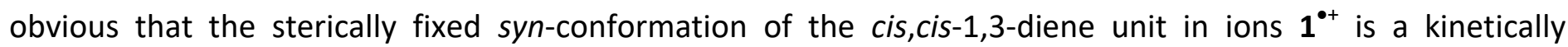
favorable feature for the cyclization process and for the subsequent loss of the methoxycarbonyl radical.

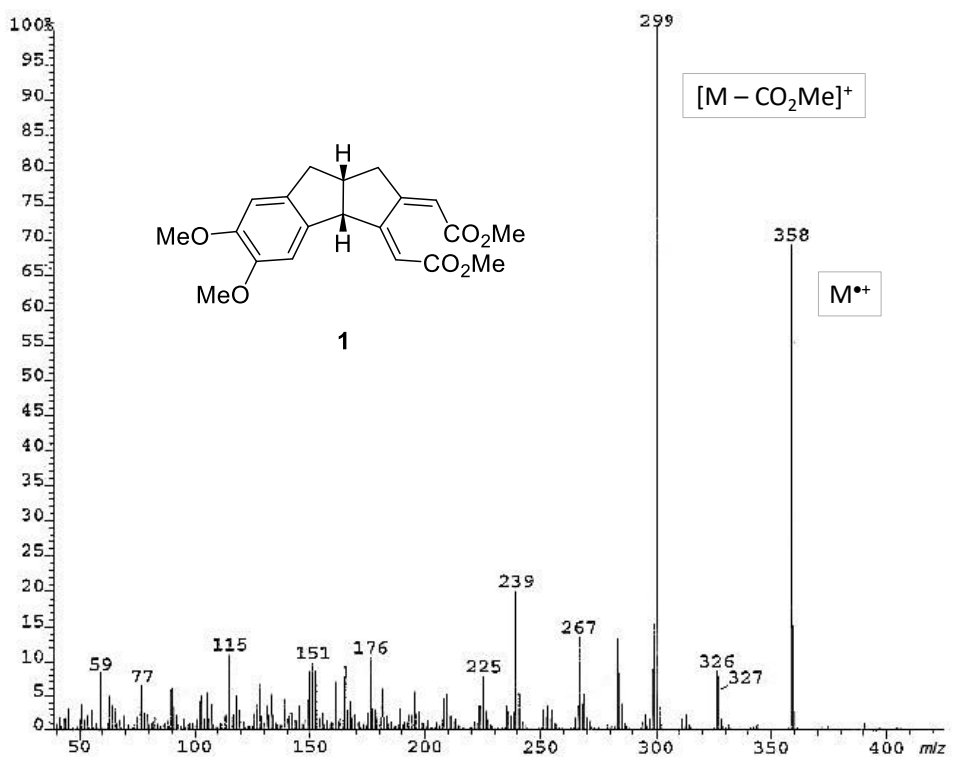

Figure 1. El mass spectrum (70 eV) of the diquinane-fused muconic acid 1.

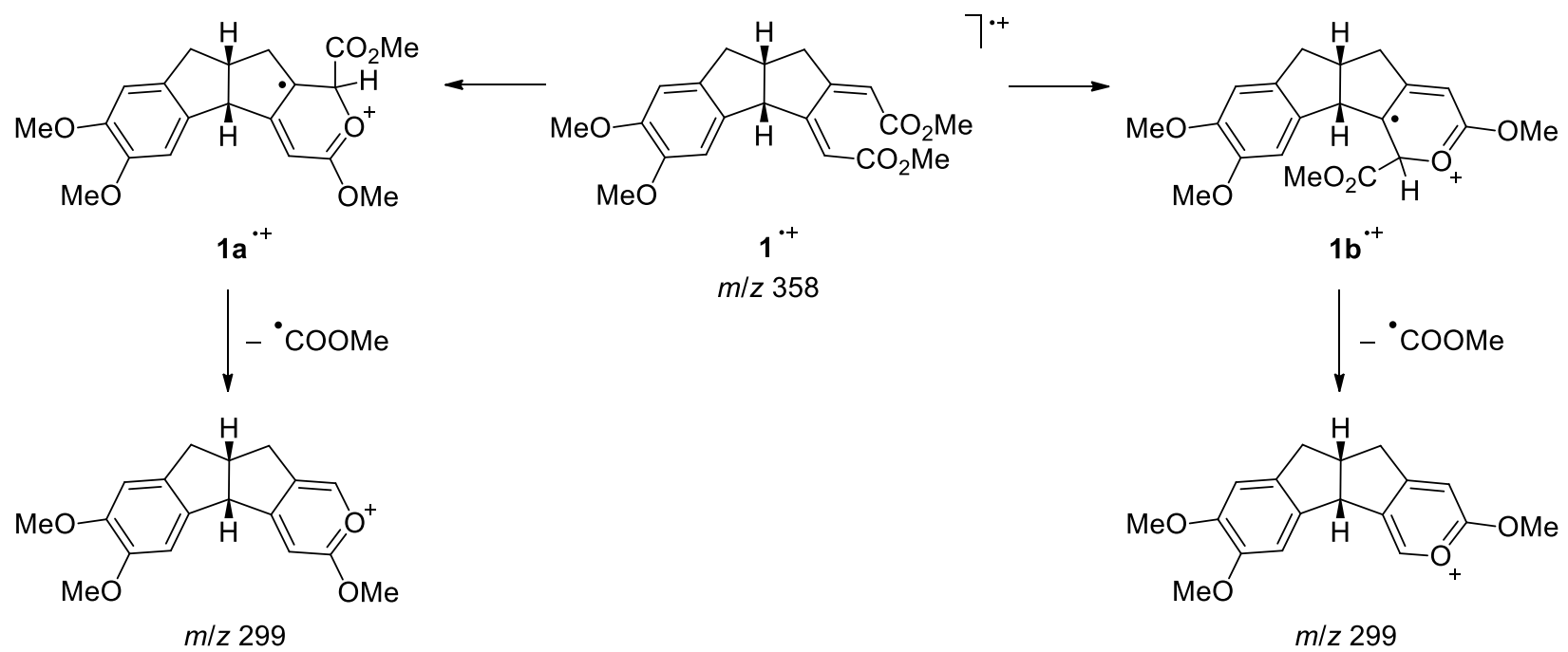

Scheme 1. Major El-induced fragmentation of the veratrolodiquinane-fused dimethyl muconate 1 by loss of a methoxycarbonyl radical (59 u). 
Two stereoisomeric dibenzotriquinane-derived dimethyl muconates, the cis,cis-isomer 2 and the cis,transisomer 3, were available by ozonolysis of the corresponding 2,3-dimethoxytriptindane ${ }^{4}$ and subsequent epimerization of the former isomer. ${ }^{12}$ Furthermore, two analogs of the single cis,cis-muconate $\mathbf{2}$, bearing either two or three dimethyl cis,cis-muconate groupings fused to a [3.3.3]propellane core, $\mathbf{4}$ and 5, were prepared by ozonolysis from the respective tetra- and hexamethoxytriptindanes (Figure 2 ). ${ }^{4}$

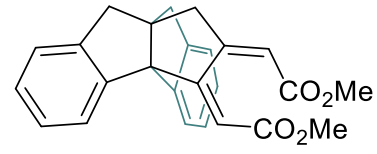

2

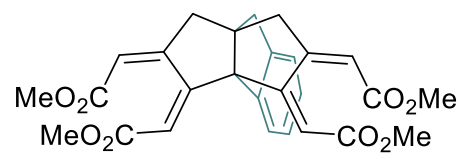

4

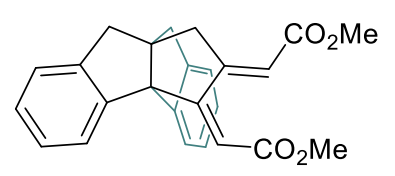

3

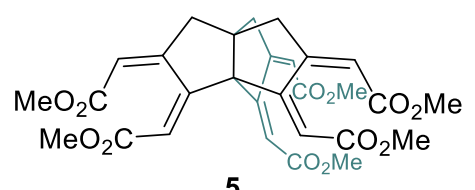

5

Figure 2. The stereoisomeric dibenzo[3.3.3]propellane-fused dimethyl muconates $\mathbf{2}$ and $\mathbf{3}$ and the bis- and triscis,cis-muconate congeners $\mathbf{4}$ and $\mathbf{5}$.
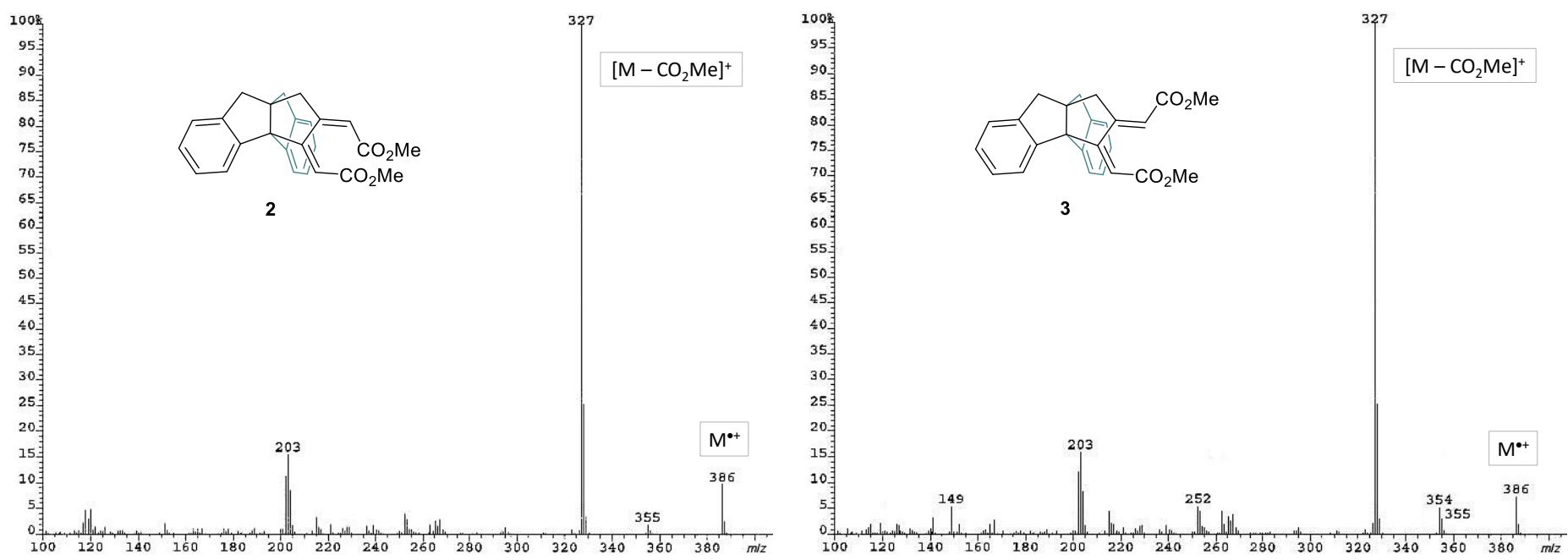

Figure 3. El mass spectra (70 eV) of the stereoisomeric triquinane-fused dimethyl muconates $\mathbf{2}$ and $\mathbf{3}$.

Similar to the diquinane muconate 1, the El mass spectra of the triquinane- (or [3.3.3]propellane-) fused analogs $\mathbf{2}$ and $\mathbf{3}$ are dominated by the corresponding [M - COOMe] fragment ion peak ( $\mathrm{m} / z$ 327) (Figure 3). Elimination of methanol is absent in the case of the cis,cis-isomer $\mathbf{2}$ - only a very minor loss of a methoxy radical is observed - but it does occur with small but significant abundance at $m / z 354(5 \%)$ in the case of the cis,trans-isomer 3. This feature can be attributed to the stereochemical difference between the cis,cis- and cis,trans-diene units in $\mathbf{2}$ and 3, in accordance with the characteristically different ratios $\left\{[\mathrm{M}-\mathrm{MeO}]^{+}\right\}:\{[\mathrm{M}-$ $\left.\mathrm{MeOH}]^{\bullet+}\right\}$ observed in the El mass spectra of the acyclic cis,cis- and cis,trans-3,4-diethylmuconic acid dimethyl esters. ${ }^{5}$ Thus, we may assume that elimination of methanol from ion $\mathbf{3}^{\bullet+}$ involves the trans-configurated acrylic ester moiety of the cis,trans-muconate unit and a hydrogen atom from the adjacent methylene group. This 
indicates that the configurational specificity of the muconate diene system in the molecular radical cations $\mathbf{2}^{\circ+}$ and $\mathbf{3}^{\circ+}$ is at least partially preserved during the fragmentation process, in analogy to the stereospecific fragmentation behavior of dimethyl muconates. ${ }^{5,6,12}$ Therefore, despite the similarity of the mass spectra of 2 and 3, the fragmentation of these two isomers should occur without significant cis-trans isomerization of the muconate $\mathrm{C}-\mathrm{C}$ double bonds. As a consequence, we assume that cyclization of the molecular ion of the cis,trans-isomer $\mathbf{3}^{\mathbf{0}^{+}}$, takes place by incorporation of the carbonyl group of the acrylic ester moiety adjacent to benzhydrylic bridgehead of the [3.3.3] propellane framework, as depicted in Scheme 2.

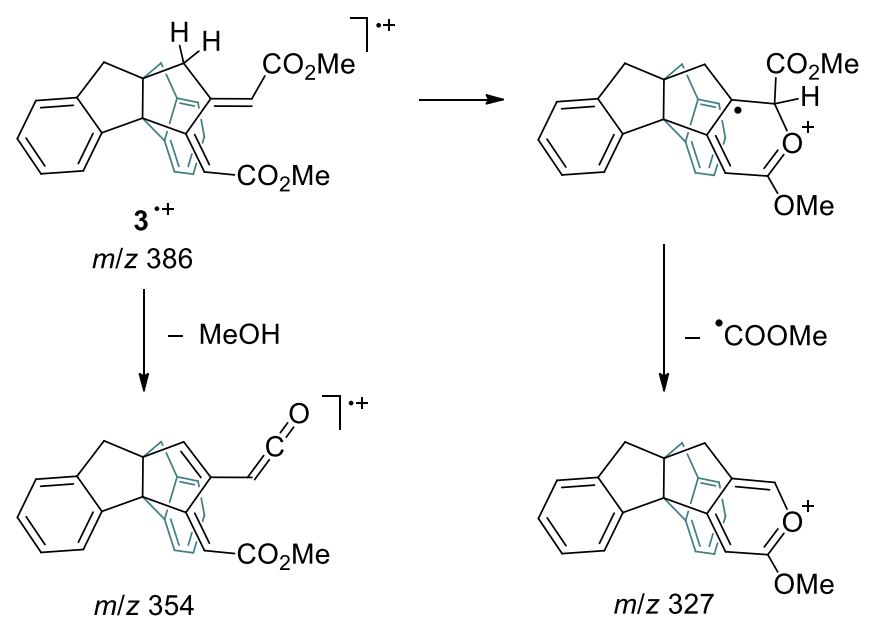

Scheme 2. El-induced fragmentation of the [3.3.3]propellane-fused dimethyl cis,trans-muconate 3 by regioselective elimination of methanol (32 u) and regioselective loss of a methoxycarbonyl radical (59 u).

The El mass spectra of the bis- and tris-(dimethyl muconates) $\mathbf{4}$ and $\mathbf{5}$ essentially reflect the same primary fragmentation behavior of the respective molecular ions (Figure 4). In the case of the lower congener, the molecular ion $4^{\bullet+}(\mathrm{m} / \mathrm{z} 478)$ undergoes exclusively the loss of a methoxycarbonyl radical, giving rise to the fragment ion with $\mathrm{m} / \mathrm{z}$ 419. The molecular ion of the higher congener, $\mathbf{5}^{\circ+}(\mathrm{m} / \mathrm{z} 570)$, behaves similarly but also loses a methoxy radical with low relative abundance to give ions with $\mathrm{m} / z 539$. In this case there is a minor elimination of methanol from the $\left[\mathrm{M}-\mathrm{COOMe}^{+}\right.$fragment ions $(\mathrm{m} / \mathrm{z} 511)$, leading to ions with $\mathrm{m} / \mathrm{z} 479$.
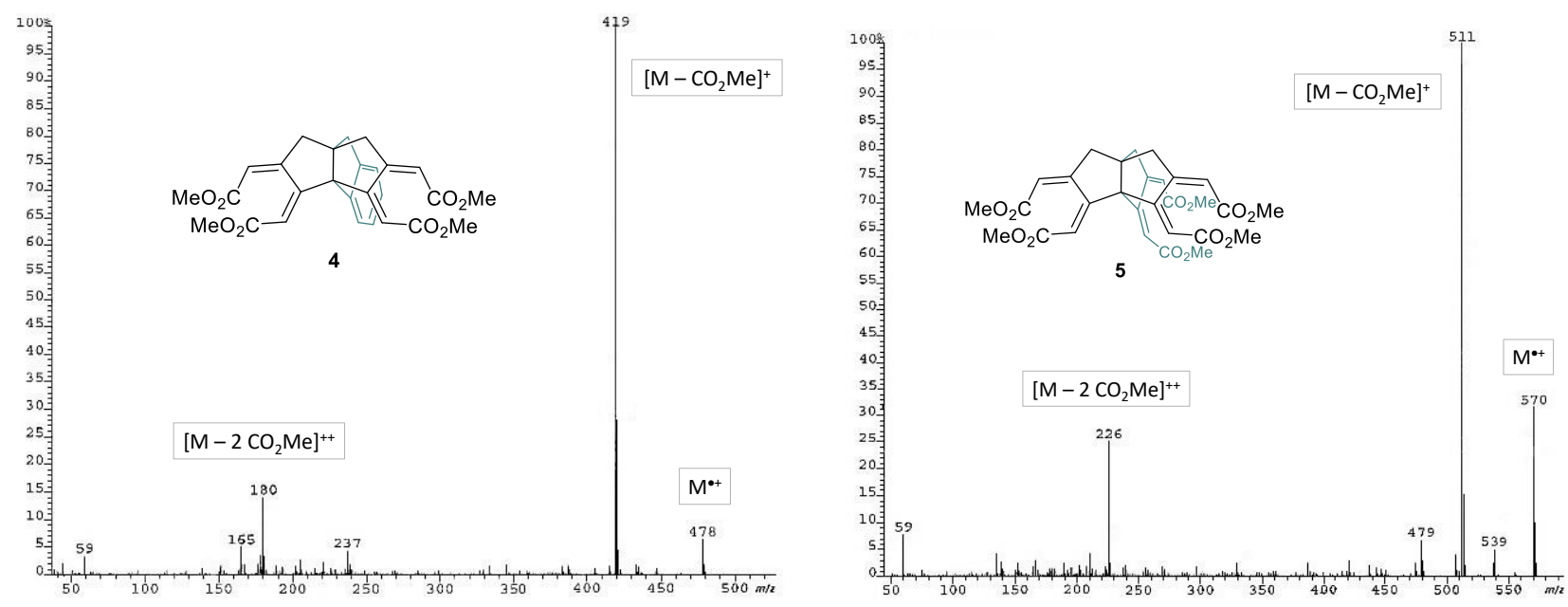

Figure 4. El mass spectra (70 eV) of the [3.3.3]propellane-fused bis-cis,cis- and tris-cis,cis-muconates 4 and $\mathbf{5}$. 
However, the mass spectra of the dimethyl muconates $\mathbf{4}$ and $\mathbf{5}$, as well as those of further bis- and tris(dimethyl muconates) presented below, reveal an additional, and quite surprising fragmentation channel. The peak at $\mathrm{m} / \mathrm{z} 180$ in the spectrum of bis-muconate $\mathbf{4}$ and that at $\mathrm{m} / \mathrm{z} 226$ in the spectrum of tris-muconate 5, both being of moderate relative intensity, do not correspond to a straightforward fragmentation of the respective molecular radical cations, $\mathrm{M}^{\bullet+}$, producing singly charged fragment ions. Rather, these peaks indicate the formation of doubly charged fragment ions formed by loss of two methoxycarbonyl radicals. In fact, closer inspection of the spectra reveals the occurrence of naturally occurring ${ }^{13} \mathrm{C}_{1}$-isotopologs at $0.5 \mathrm{~m} / \mathrm{z}$ units above the nominal mass of the fragment ions. The $[\mathrm{M}-2 \text { COOMe }]^{2+}$ ions should be formed directly from the doubly charged molecular ions of the esters, $4^{2+}(\mathrm{m} / \mathrm{z} 239)$ and $5^{2+}(\mathrm{m} / \mathrm{z} 285)$, which, however, do not appear as such in the mass spectra. This assumption corresponds nicely with the finding that the ion $[\mathrm{M}-2 \mathrm{COOMe}]^{2+}(\mathrm{m} / z$ 226) originating from tris-muconate 5 is formed with higher relative abundance (25\%) as compared to the ion $[\mathrm{M}-2 \text { COOMe }]^{2+}(\mathrm{m} / \mathrm{z} 180)$ generated from the bis-muconate $4(\mathrm{~m} / \mathrm{z} 180,15 \%)$. Loss of the second methoxycarbonyl radical by second ionization of the singly charged [M - COOMe] ${ }^{\bullet+}$ ions of $4(\mathrm{~m} / z$ 419) and $\mathbf{5}$ $(\mathrm{m} / z \mathrm{511})$ is ruled out due to the low probability of this process. In fact, the corresponding doubly charged fragment ions, [M - COOMe] $]^{0++}$, of $4(\mathrm{~m} / \mathrm{z} 209.5)$ and $5(\mathrm{~m} / \mathrm{z} 255.5)$ are not observed. It appears that the mutually isolated $\pi$-electron systems present in the ionic species generated from the neutral precursors 4 and 5 allow efficient formation of doubly charged molecular ions. As a quite intriguing consequence, we suggest that the $[\mathrm{M}-2 \mathrm{COOMe}]^{2+}$ ions generated from compounds $\mathbf{4}$ and $\mathbf{5}$ both bear two mutually separated pyrylium ion units which, due to their aromatic character and in spite of the two relatively close positive charges within the same ionic species, provide sufficient stability. The fragmentation paths leading to the singly charged fragment ions $\left[\mathrm{M}-\mathrm{COOMe}^{\circ+}\right.$ and the corresponding dications $[\mathrm{M}-2 \mathrm{COOMe}]^{2+}$ and their tentative structures are depicted in Scheme 3 for the case of the [3.3.3]propellane-fused bis-muconate 4 . Admittedly, the survival of the propellane core is tentative. To the best of our knowledge, no bis-pyrylium salt containing two such cationic heteroaromatic units fused to the very same propellane framework is known to date, neither in the gas phase nor in condensed matter.

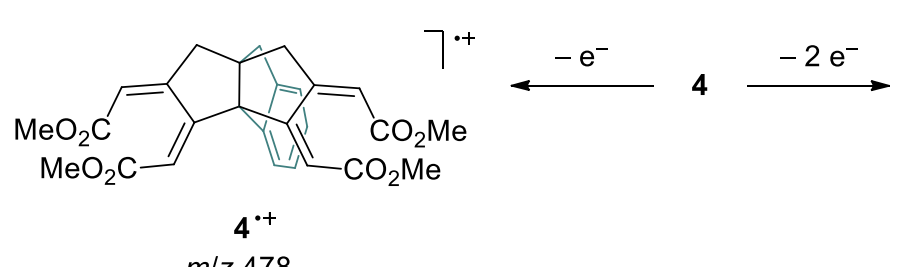

$m / z 478$
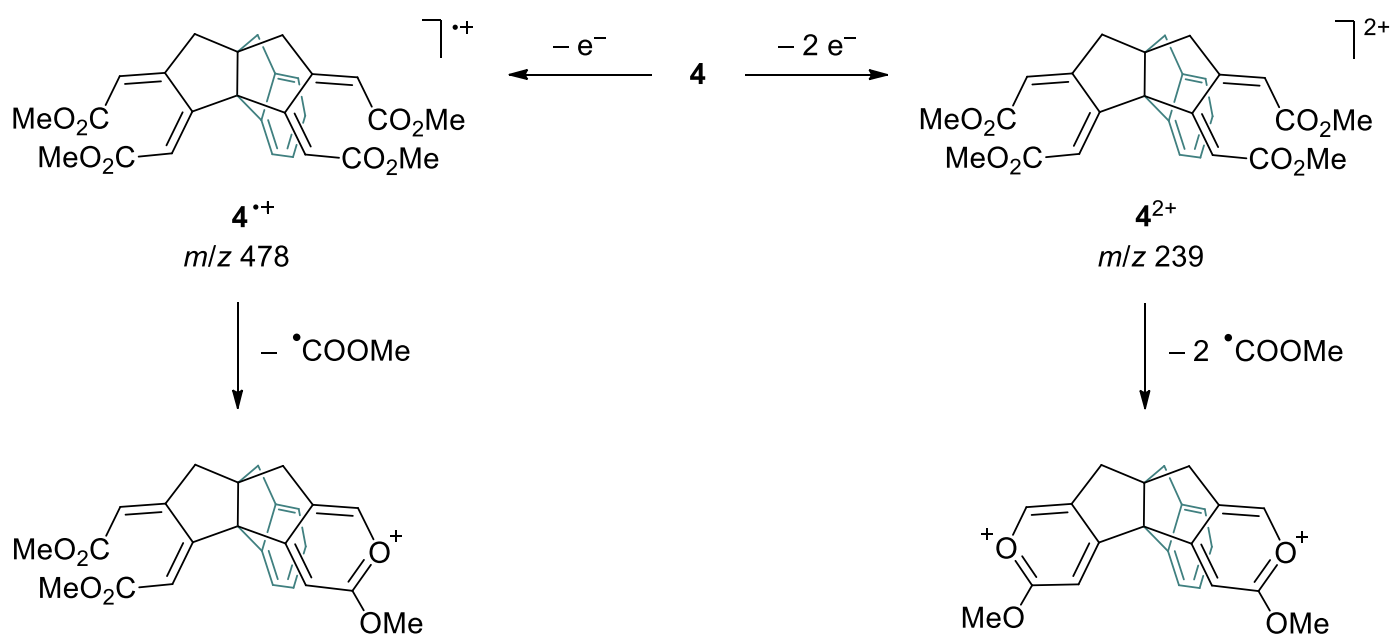

/z 239

$m / z 419$

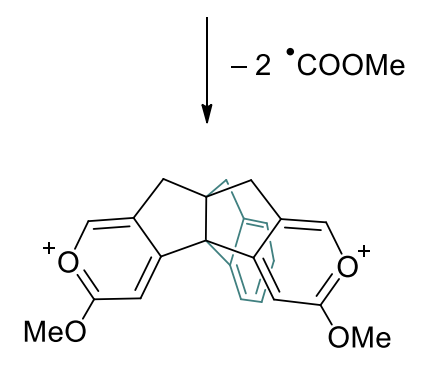

$m / z 180$

Scheme 3. El-induced loss of a methoxycarbonyl radical ( $59 \mathrm{u}$ ) from the molecular radical cation of the [3.3.3]propellane-fused bis-muconate 4 and loss of two methoxycarbonyl radicals from the doubly charged molecular cation $4^{2+}$, giving rise to mono- and bis-pyrylium ions, respectively. Only one of the possible isomeric fragment ions is shown in each case. 


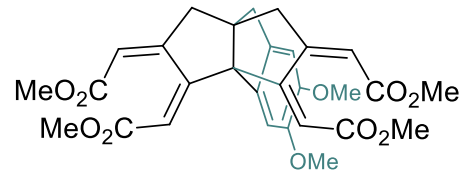

6

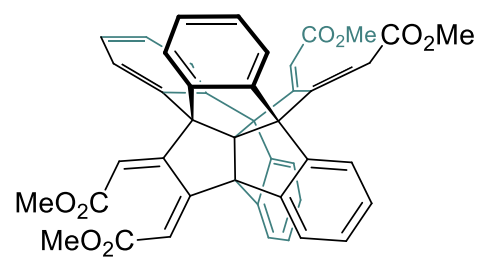

8

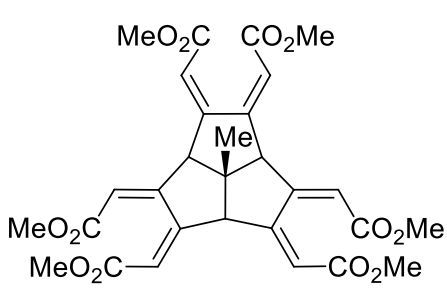

7

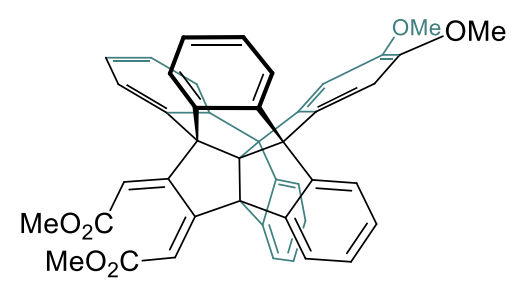

9

Figure 5. The veratrolo[3.3.3]propellane-fused bis-cis,cis-muconate 6, the triquinacene-based congener 7 and the centrohexaquinane-fused bis- and mono-cis,cis-muconates $\mathbf{8}$ and $\mathbf{9}$.

As mentioned above, the surprising loss of two methoxycarbonyl radicals from the doubly charged molecular ions was also observed with a number of other centropolyquinane-fused bis- and tris-(dimethyl muconates), 6-8 (Figure 5). ${ }^{3,4,13}$ These include a product of the ozonolytic degradation of veratrole analogs of a tribenzotriquinacene derivative and of centrohexaindane.,4 The fragmentation of the four triquinane-fused congeners is displayed in Scheme 4. Besides the [3.3.3]propellane derivatives 4-6, the hexahydrotriquinacenefused tris-muconate 7 shows the same fragmentation behavior, albeit to a relatively low extent. ${ }^{14}$ One may speculate that the presence of the particularly electron-rich veratrole ring increases the stability of the molecular ion $6^{\circ+}$ as compared to the less electron-rich ion $4^{\circ+}$. In any case, however, the corresponding [M - 2 $\mathrm{COOMe}^{2+}$ fragment ions, representing bis-pyrylio-veratrolo- and benzo-bis-pyrylio-fused[3.3.3]propellanes, respectively, should be rather stable dicationic species. The chemistry of the corresponding parent tribenzo[3.3.3]propellane, triptindane, has been developed since $1966 .{ }^{3,15,16}$ It is worth noting that no further fragmentation of the dications $[\mathrm{M}-2 \mathrm{COOMe}]^{2+}$ occurs in any of the cases observed here. In fact, heterolytic $\mathrm{C}-\mathrm{C}$ bond cleavage of the [3.3.3]propellane framework would destroy the aromatic character of the two wellstabilized pyrylium units. The fragmentation of the two isomeric tris-(dimethyl muconates) 5 and $\mathbf{7}$ leads to similar assumptions. In these cases, the third muconate unit that persists in the $[\mathrm{M}-2 \mathrm{COOMe}]^{2+}$ ions does not suffer any further decomposition. Thus, the presence of the formally neutral aromatic ring in the [M -2 $\mathrm{COOMe}^{2+}$ ions formed from the bis-muconates $\mathbf{4}$ and $\mathbf{6}$ is not essential but, evidently, the presence of at least two muconate units in the neutral precursors is. However, tricationic fragment ions formed by threefold loss of ${ }^{\bullet}$ COOMe, which might be insinuated for the tris-muconates $\mathbf{5}$ and $\mathbf{7}$, are not observed. 

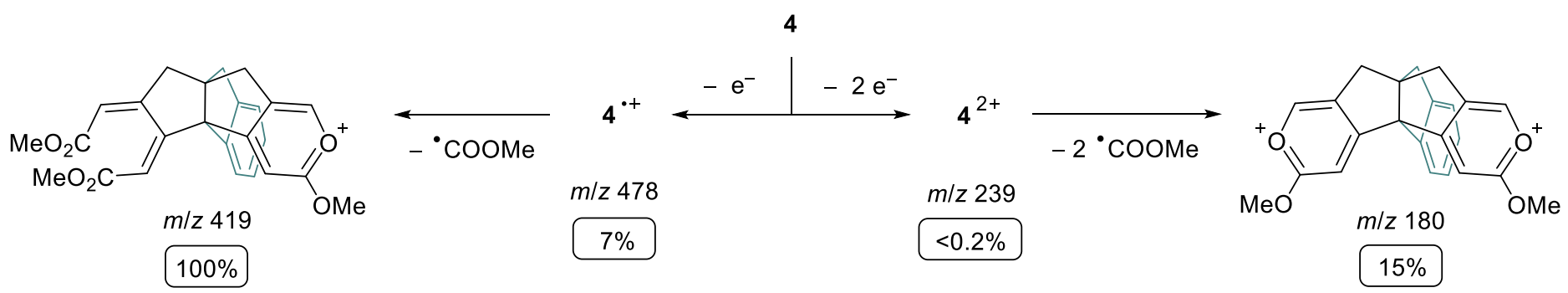

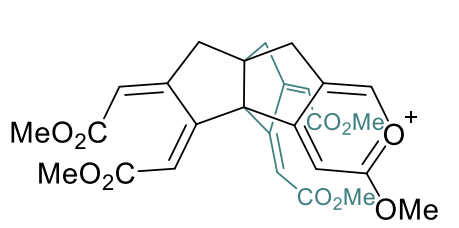

$m / z 511$

$100 \%$

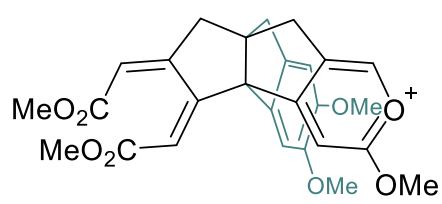

$\mathrm{m} / \mathrm{z} 479$

$100 \%$

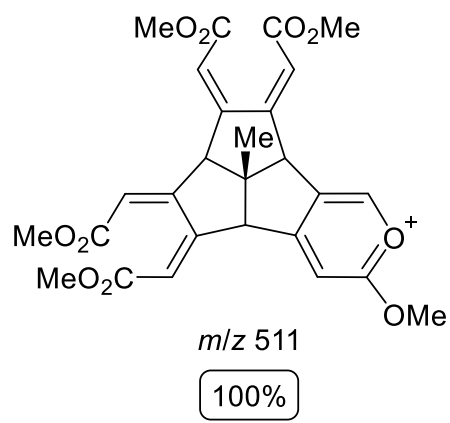

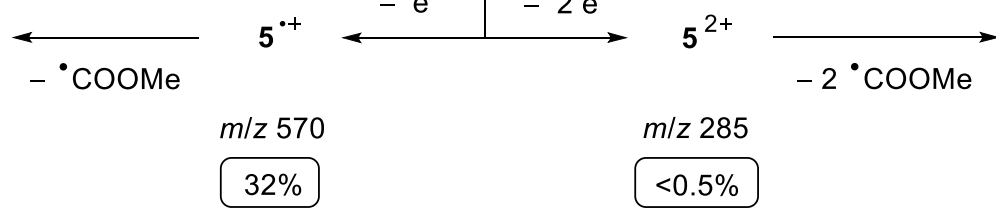
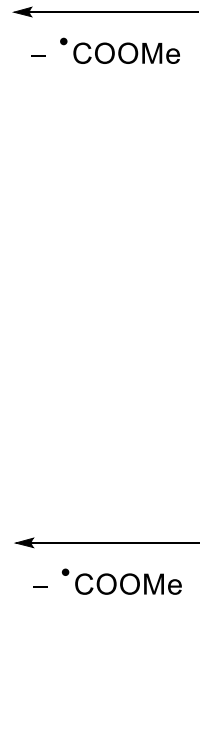

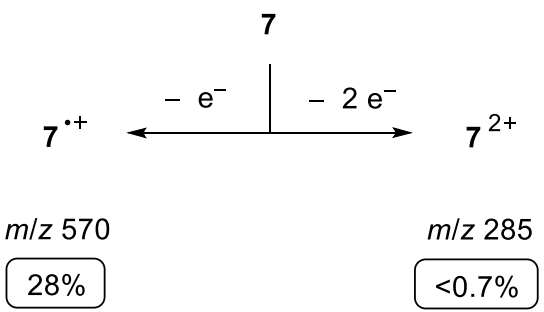

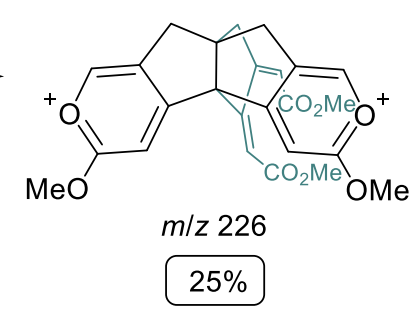
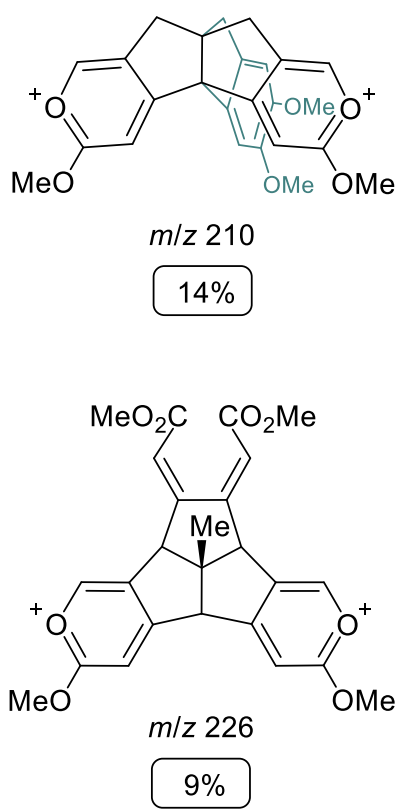

Scheme 4. Loss of one and two methoxycarbonyl radicals from the molecular radical cations and, respectively, from the molecular dications of the triquinane-fused bis- and tris-muconates 4-7. The relative abundances of the ions are framed. In each case, only one of the three possible isomers of the fragment ions is shown. Note: The $m / z 239$ peak (2\%) in the mass spectrum of compound 4 is due to a singly charged ion (see Figure 4).

Finally, the fragmentation of the two centrohexaquinane-fused congeners $\mathbf{8}$ and $\mathbf{9}$ (Figure 5) deserves to be presented here (Scheme 5). The ozonolytic degradation of the corresponding tetramethoxycentrohexaindane ${ }^{4,17,18}$ yielded tetraester $\mathbf{8}$, a fenestrindane derivative bearing two bridging dimethyl cis,cismuconates, and the related diester 9, a centrohexaquinane derivative in which one veratrole unit has survived. The El mass spectrum of the bis-muconate 8 exhibits the same fragmentation behavior as do those of the lower congeners 4-7. Again, loss of a methoxycarbonyl radical gives rise to the base peak at $m / z 641$ and two such radicals are lost after twofold ionization, generating the ion $[\mathrm{M}-2 \mathrm{COOMe}]^{2+}(\mathrm{m} / \mathrm{z} 291)$ with remarkably high relative abundance (42\%). This fragment ion is particularly noteworthy because it represents the first heteroaromatic analog of centrohexaindane carrying two positive charges at the centrohexacyclic, 
topologically non-planar framework..$^{3,17,18}$ The synthesis of the corresponding three-dimensional bis-pyrylium salts should be possible.

The centrohexaquinane-fused derivative 9, representing a simple dimethyl muconate akin to the lower congeners 1-3, again undergoes the loss of a methoxycarbonyl radical as the strongly dominant fragmentation, giving rise to ions with $\mathrm{m} / \mathrm{z} 609$ (Scheme 5). However, the El mass spectrum of 9 also exhibits the formation of a doubly charged species: There is no doubt that the peak at $\mathrm{m} / \mathrm{z} 304.5$ of moderate intensity $(17 \%)$ is due to a radical dication, namely $[\mathrm{M}-\mathrm{COOMe}]^{0++}$. This highly unusual ion is formed by cleavage of the molecular dication $\mathbf{9}^{2+}(\mathrm{m} / \mathrm{z} 334)$ under single cyclization, as discussed above, while the veratrole unit carrying the second charge remains intact. It is worth noting that the $\left[\mathrm{M}-2 \mathrm{COOMe}^{2+}\right.$ ions generated from the bisand tris-muconates 4-8 all represent distonic dications, with the two positive charges residing in isolated $\pi$ electron systems, whereas the $\left[\mathrm{M}-\mathrm{COOMe}^{\bullet++}\right.$ ion with $\mathrm{m} / \mathrm{z} 304.5$ formed from the mono-muconate 9 represents a "conventional" distonic radical cation. ${ }^{19}$ Knowledge on distonic dications of polyaromatic structures is quite scarce, in contrast to distonic dications formed by protonation of bioorganic oligomers and polymers.

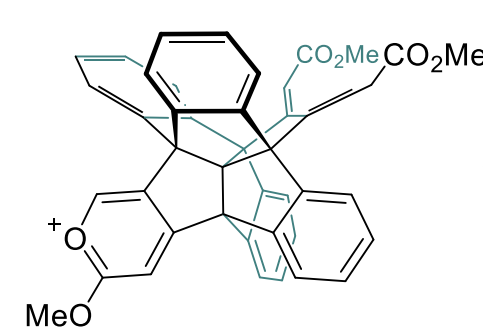

$\mathrm{m} / \mathrm{z} 641$ $100 \%$

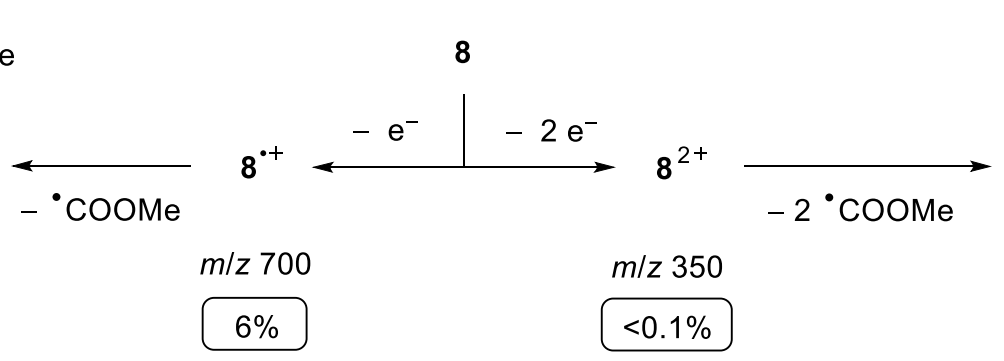

$6 \%$

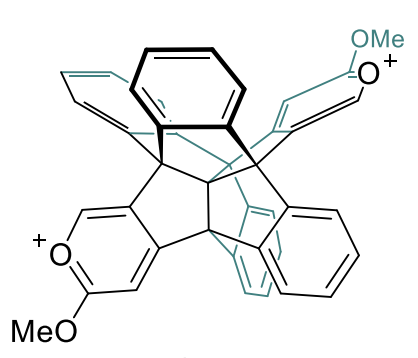

$\mathrm{m} / \mathrm{z} 291$

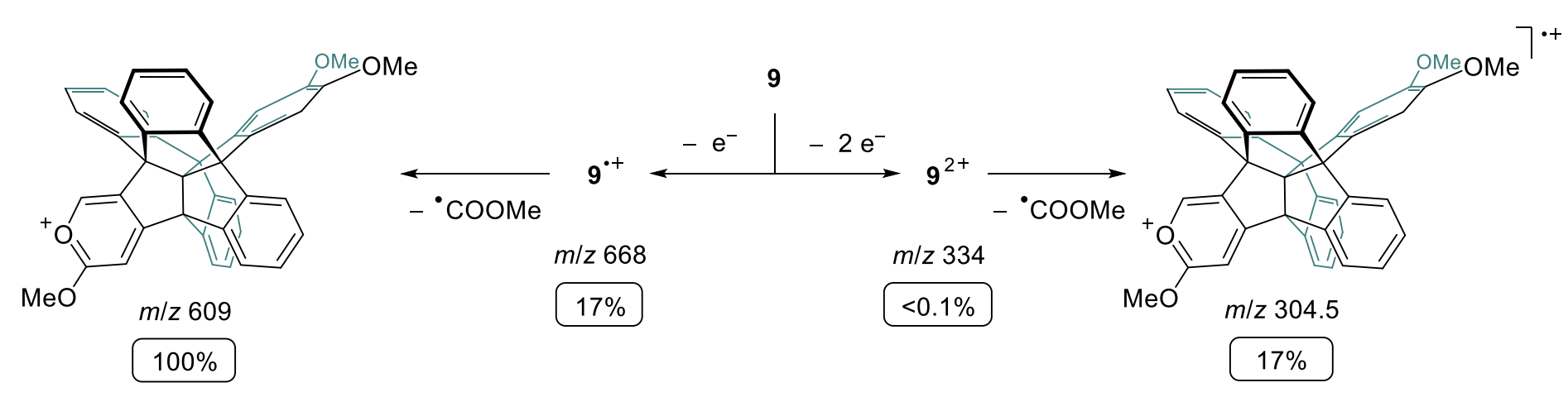

Scheme 5. Top: Loss of one and two methoxycarbonyl radicals from the molecular radical cation and, respectively, from the molecular dication of the hexaquinane-fused bis-muconate $\mathbf{8}$. Bottom: Loss of a single methoxycarbonyl radical from both the molecular radical cation and the molecular dication of the hexaquinane-fused mono-muconate 9. The latter fragmentation gives rise to the distonic radical dication $[\mathrm{M}-\mathrm{COOMe}]^{\bullet++}(\mathrm{m} / \mathrm{z}$ 304.5). The relative abundances of the ions are framed. 


\section{Conclusions}

This report describes the unusual mass spectrometric fragmentation behavior of unusual organic compounds, thus expanding our understanding based on the known fragmentation behavior of rather common compounds. It confirms known mechanistic understanding and widens our view based on unexpected observations. It is demonstrated that various muconic acid dimethyl esters bearing one, two or even three cis,cis-muconate units fused to a rigid polycyclic di-, tri- or hexaquinane core with a fixed mutual synorientation of the two ester groups undergo the characteristic loss of a methoxycarbonyl radical from the molecular radical cations. This characteristic reaction involves cyclization of the ionized muconate group generating an ionized $\alpha$-pyran ring and subsequent loss of the methoxycarbonyl residue from the radicalcationic intermediate. While this isomerization-dissociation sequence is in complete accordance to the wellknown fragmentation of simpler dimethyl muconates and other carbonyl compounds that bear $\alpha, \gamma$-diene unsaturation, it is remarkable as such because of the strong dominance in the El mass spectra.

However, when two or three muconate groups are present in these polycyclic muconic acid dimethyl esters, the occurrence of doubly charged fragment ions formed after the loss of two methoxycarbonyl radicals is observed in high relative abundance and throughout the whole series. The molecular dications, $\mathrm{M}^{2+}$, and the doubly charged primary fragment ions, $\left[\mathrm{M}-\mathrm{COOMe}^{\circ++}\right.$, both not being observed in the mass spectra of the bis- and tris-muconates, must play an important role in the production of the unusual but highly characteristic $[\mathrm{M}-2 \mathrm{COOMe}]^{2+}$ ions. In one single case, the $\left[\mathrm{M}-\mathrm{COOMe}^{0++}\right.$ ions were observed in considerable abundance. It is obvious to postulate the structure of the $[\mathrm{M}-2 \mathrm{COOMe}]^{2+}$ ions: The polycyclic cores of the neutral precursors should have remained intact and bear two aromatic pyrylium rings, resulting in rigid, polycyclic dipyrylio-annelated [3.3.3]propellane- and fenestrindane-based distonic dications.

Mass spectrometry and organic chemistry - may this interplay never fade away! $!^{20}$

\section{Experimental Section}

The El mass spectra were recorded by use of an Autospec $X$ sector-field mass spectrometer with EBE geometry (Fisons, Manchester, UK) equipped with a standard El ion source. The samples were evaporated from aluminum crucibles introduced into the source by a solids probe. Electron energy $70 \mathrm{eV}$, accelerating voltage 8000 V. The OPUS software (V3.6, Micromass 1998) was used for processing the data. For further details, see ref. 4.

\section{References}

1. Maccoll, A. Org. Mass Spectrom. 1968, 1, 1-1.

http://dx.doi.org/10.1002/oms.1210010102

2. Kuck, D. Chem. Rec. 2015, 15, 1075-1109.

http://dx.doi.org/10.1002/tcr.201500023

3. Kuck, D. Chem. Rev. 2006, 106, 4885-4925.

http://dx.doi.org/10.1021/cr050546+

4. Harig, M.; Neumann, B.; Stammler, H. G.; Kuck, D. ChemPlusChem 2017, 82, 1078-1095.

http://dx.doi.org/10.1002/cplu.201700090 
5. Gil-Av, E.; Leftin, J. H.; Mandelbaum, A.; Weinstein, S. Org. Mass Spectrom. 1970, 4, 475-479. http://dx.doi.org/10.1002/oms.1210040148

6. Mandelbaum, A.; Weinstein, S.; Gil-Av, E.; Leftin, J. H. Org. Mass Spectrom. 1975, 10, 842-849. http://dx.doi.org/10.1002/oms.1210101006

7. Schaldach, B.; Grützmacher, H. F. Int. J. Mass Spectrom. Ion Phys. 1979, 31, 257-270. http://dx.doi.org/10.1016/0020-7381(79)83027-2

8. Schaldach, B.; Grützmacher, H. F. Int. J. Mass Spectrom. Ion Phys. 1979, 31, 271-286. http://dx.doi.org/10.1016/0020-7381(79)83028-4

9. Schaldach, B.; Grützmacher, H. F. Org. Mass Spectrom. 1980, 15, 175-181. http://dx.doi.org/10.1002/oms.1210150404

10. Schaldach, B.; Grotemeyer, B.; Grotemeyer, J.; Grützmacher, H. F. Org. Mass Spectrom. 1981, 16, 410-415. http://dx.doi.org/10.1002/oms.1210160911

11. Schaldach, B. Int. J. Mass Spectrom. Ion Processes 1984, 56, 237-241. http://dx.doi.org/10.1016/0168-1176(84)85051-X

12. Harig, M. Doctoral Thesis, Bielefeld University, Bielefeld, 2002.

13. Gund, P.; Gund, T. M. J. Am. Chem. Soc. 1981, 103, 4458-4465. http://dx.doi.org/10.1021/ja00405a028

14. The El mass spectra of compounds $4-7$ and 9 are reproduced in ref. 4 . Numeric data of the mass spectra of these compounds and of compound $\mathbf{8}$ can also be found there.

15. Thompson, H. W. J. Org. Chem. 1968, 33, 621-625. http://dx.doi.org/10.1021/jo01266a032

16. Thompson, H. W. Tetrahedron Lett. 1966, 7, 6489-6494. http://dx.doi.org/10.1016/S0040-4039(00)76131-4

17. Kuck, D.; Linke, J.; Teichmann, L. C.; Barth, D.; Tellenbröker, J.; Gestmann, D.; Neumann, B.; Stammler, H. G.; Bögge, H. Phys. Chem. Chem. Phys. 2016, 18, 11722-11737. http://dx.doi.org/10.1039/C5CP07005H

18. Linke, J.; Neumann, B.; Stammler, H. G.; Kuck, D. Chem. Eur. J. 2018, 24, 9316-9324. http://dx.doi.org/10.1002/chem.201801806

19. Hammerum, S. Mass Spectrom. Rev. 1988, 7, 123-202. http://dx.doi.org/10.1002/mas.1280070202

20. Firmino, T. R.; Riveros, J. M. New J. Chem. 2016, 40, 54-57. http://dx.doi.org/10.1039/c5nj02250a 